\title{
La formación del profesorado ante las TIC
}

\section{Paula Morales Almeida, Universidad de Las Palmas de Gran Canaria, Gran Canaria-Las Palmas, España}

\begin{abstract}
Resumen: La sociedad de la información y la comunicación ha traído consigo las tecnologías de la sociedad y la información (en adelante TIC), lo que ha conllevado muchos cambios, sobre todo en educación. En esta última se aprecian dichos cambios tanto en los recursos; ordenadores, pizarras digitales, como en el rol que ejerce el profesor ante esta nueva situación. El docente ha visto cambiado su papel; de ser el transmisor del conocimiento ha pasado a ser guía de sus alumnos; de ser protagonista, a quedar relegado a un segundo plano. Es por ello que esta nueva sociedad necesita de profesores formados y motivados para poder adaptar la escuela a esta nueva realidad. No se trata de apartar lo anterior, de cambiar totalmente la metodología, sino de incluir lo mejor de la tecnología y utilizarla de apoyo para mejorar el proceso de enseñanza-aprendizaje. Para ello se necesitan los recursos adecuados, ya que sin ellos es imposible que se dé un cambio real. Las TIC ofrecen multitud de posibilidades en educación. Es deber del docente conocer dichas posibilidades y adaptarlas a su trabajo con los alumnos, ya que así se conseguirán ciudadanos plenamente integrados en la sociedad 2.0 y que a su vez, ejerzan un uso responsables de las TIC. Para conocer la formación del profesorado, así como sus motivaciones ante esta nueva realidad, se ha procedido a realizar un cuestionario a un grupo de profesores de secundaria. Con los resultados obtenidos se podrán conocer cuáles son sus pensamientos sobre las TIC y de qué manera piensan utilizarlas en su trabajo diario con los alumnos. Así, se podrán saber las limitaciones que existen con respecto a este tema y hacerle frente.
\end{abstract}

Palabras Clave: Formación Inicial, Profesorado, TIC, Aprendizaje, Alumnado.

Abstract: The information society has developed and communication technologies and the information society (ICT), which has brought many changes, especially in education. In education, these changes are seen in both resources; computers, digital blackboard, and in the role exerted by the teacher. The teacher has seen his role changed, to be the transmitter of knowledge has become guide their pupils, to be protagonist to be relegated to the background. That is why this new society needs trained and motivated teachers to adapt the school to this new reality. This is not the above aside, completely change the methodology, but to include the best of the technology and use of support to improve the teaching-learning process. This requires adequate resources, because without them it is impossible to give a real change. ICT offers a multitude of possibilities in education. It is the duty of the teacher to know those opportunities and adapt to their work with students, as this will get fully integrated citizens in society 2.0 and that in turn, exert a responsible use of ICT. For teacher training and their motivations in this new reality, we have proceeded to conduct a questionnaire to a group of secondary teachers. With the results obtained may know what your thoughts on ICT and how they intend to use in their daily work with students. So, you may know that there are still limitations with respect to ICT.

Keywords: Initial Training, Teachers, ICT, Learning, Students

TEXTOS. Revista Internacional de Aprendizaje y Cibersociedad

Volumen 17, Número 1, 2013, http://aprendizaje-cibersociedad.com/journal, ISSN 1577-3760

(C) Common Ground España, Paula Morales Almeida, Todos los Derechos Reservados, Permisos:

cg-support@commongroundpublishing.com 


\section{Formación del Profesorado}

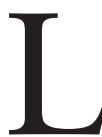

A SOCIEDAD DE la información ha generado muchos cambios en todos los ámbitos de la vida y la educación no podía ser menos. Uno de los cambios experimentados es el rol del profesor. Hasta hace poco, el docente era considerado el protagonista de la enseñanza, ya que era él quien transmitía todos sus conocimientos a sus alumnos y estos eran meros receptores, los cuales no podían aprender sin su maestro. Actualmente se busca a un profesor que actúe más como guía, como orientador del proceso formativo de sus alumnos. El protagonismo lo tienen ahora los discentes, quienes son los verdaderos protagonistas de su proceso de enseñanza-aprendizaje. El propio Cabero (2004) señala la importancia de que el profesor cambie de rol y guíe al alumno hacia su aprendizaje aportándole todas las herramientas que necesite para que llegue a ese fin. Barberá (2003: 60) comenta que, "en la sociedad de la información, el profesor deja de ser considerado el único poseedor de un saber que sólo tendría que transmitir. Ahora se convierte fundamentalmente en el asociado de un saber colectivo que debe organizar y ayudar a compartir. En esta perspectiva, el proceso de enseñanza y aprendizaje se ve como un diálogo en colaboración entre elementos diversos entre los cuales destaca la voz del profesorado por su capacidad de estructurar, facilitar y guiar esta interacción". Por lo tanto, no se habla de saber individual sino de saber colectivo, por el bien de la comunidad, protagonizada por los estudiantes. Cuando el profesor haya admitido el nuevo rol que le toca interpretar en esta nueva sociedad avanzaremos hacia una total integración de las TIC en las escuelas. Pero no será suficiente con admitir dicho rol, sino que la formación toma una gran importancia en todo este proceso.

Así, ¿por qué es necesario que el profesor se forme en materia de tecnologías de la información y la comunicación (TIC)? Según Cabero (2004: 2) "dos son los aspectos que hacen más necesaria esta formación: uno, la diversidad de funciones que empiezan a desempeñar estas TIC, y dos, su impacto en variables críticas de los procesos de enseñanza-aprendizaje, entre ellas, sobre los diferentes roles que el profesor desempeñará en los mismos". Con respecto al primero, las TIC desempeñan diversas funciones dentro de la educación; como apoyo al aprendizaje presencial, como fuente de información, como red social y por otro lado, en relación al segundo punto, los procesos de enseñanza-aprendizaje han cambiado y con ellos el rol del profesor. Es por ello que se considera la formación en TIC esencial para poder estar integrado en la sociedad de la información y la comunicación y es un tema que ningún docente puede dejar pasar, ya que aquel que no recibe dicha formación se habrá quedado rezagado con respecto a sus propios compañeros pero también frente a la sociedad en general, ya que las TIC no las encontramos solo en la educación, sino en todos los ámbitos de la vida. Tampoco podemos olvidar que la tecnología se encuentran en permanente cambio y cada día asistimos a nuevas formas de comunicación y los alumnos, por su edad y motivación, son las primeras personas que se encuentran al día en dicha materia, otra razón más para que los docentes se formen también y acompañen a sus alumnos en este nuevo tipo de aprendizaje.

Las TIC nos ofrecen múltiples posibilidades entre las que Cabero (2004) destaca:

- Eliminar las barreras espacio temporales entre el profesor y el estudiante y por tanto servir de ampliación a los escenarios analógicos y virtuales de formación.

- Flexibilizar la enseñanza, tanto en lo que respecta al tiempo, al espacio, a las herramientas de comunicación, como a los códigos con los cuales los alumnos desean interaccionar. 
- Ampliar la oferta formativa para el estudiante.

- Favorecer la creación de escenarios tanto para el aprendizaje cooperativo como para el autoaprendizaje.

- Posibilitar el uso de herramientas de comunicación sincrónicas y asincrónicas.

- Potenciar el aprendizaje a lo largo de toda la vida.

- Favorecer la interacción e interconexión de los participantes en la oferta educativa.

- Adaptar los medios a las necesidades, características, estilos de aprendizaje e inteligencia múltiples de los sujetos.

- Ayudar a comunicarse e interaccionar con su entorno a los sujetos con necesidades educativas especiales.

- Presentar escenarios multimedia-multicódigos e hipertextual/hipermedia, que propician la utilización de escenarios convergentes de tecnologías y que los lectores se conviertan en lectoautores, y por tanto que en su utilización sean procesadores activos y constructores del conocimiento.

- Romper los clásicos escenarios formativos, limitados a las instituciones escolares.

- Y ofrecer nuevas posibilidades para la orientación y la tutorización de los estudiantes.

Es difícil negar que con las TIC podemos crear un ambiente de aprendizaje mucho más óptimo, tanto para los estudiantes como para el profesorado, ya que nos ayudará a sentirnos más cerca del alumno. Pero las TIC deben verse como un recurso, un apoyo a la enseñanza presencial y nunca sustituir a esta.

¿Cómo debe ser la formación del profesorado? Cebrián de la Cerna (2003: 35) afirma que "los profesores del futuro deben tener una serie de competencias de cara a la utilización de las TIC, y que de su análisis podemos también encontrar dimensiones para su formación", en concreto el autor nos señala los siguientes conocimientos: sobre las diferentes formas de trabajar las TIC en sus contenidos y área específica; para desarrollar enseñanza en diferentes espacios y recursos; organizativo y planificación de aula; dominio para la inserción de las técnicas y medios para la formación en cualquier espacio y tiempo que combine la formación presencial con la formación a distancia; y para la selección de materiales.

Por otra parte, tan importante como las grandes dimensiones que deben de guiar la formación del profesorado, es también asumir algunos principios que deben de dirigirla. Estos principios, según Cabero (2004: 7) son: "el valor de la práctica y la reflexión sobre la misma, contemplar problemas reales para los docentes no para los formadores o los técnicos, la participación del profesorado en su construcción y determinación, su diseño como producto no acabado, centrarse en los medios disponibles, situarse dentro de estrategias de formación más amplias que el mero audiovisualismo y el alcance en consecuencia de dimensiones más amplias como la planificación, diseño y evaluación, su desarrollo en contextos naturales de enseñanza, la utilización de la deconstrucción de mensajes mediados como principios para el aprendizaje de su realización, y la coproducción de materiales entre profesores y expertos". En definitiva, tener en cuenta que la formación va dirigida a un profesional de la enseñanza, no a ingenieros, por lo que la formación debe estar adaptada a las necesidades de los docentes. Debe ser una formación que le permita aplicarla en clase, con sus alumnos, donde vea la utilidad o no de la herramienta y conozca tanto las ventajas y desventajas de su uso en el aula. 


\section{Competencias en materia de TIC}

Aparte de estar formado, se espera que el profesor sea competente. Así, cuando hablamos de las competencias que debe tener el profesorado en materia de TIC debemos tener claro qué entendemos por competencia docente. Según Escudero (2006: 34), es el "conjunto de valores, creencias y compromisos, conocimientos, capacidades y actitudes que los docentes, tanto a título personal como colectivo (formando parte de grupos de trabajo e instituciones educativas) habrían de adquirir y en las que crecer para aportar su cuota de responsabilidad a garantizar una buena educación a todos". Vemos como Escudero va más allá de los simples conocimientos, incluye valores, creencias, capacidades que van a configurar el carácter del propio docente, que lo definen no solo como profesor sino también como persona, ya que no olvidemos que no se educa solo con lo que se dice o se sabe, sino con quien se es, con lo que se hace, con lo que se piensa. Y de la competencia docente, se pasa a la competencia digital. Según Prendes y Gutiérrez (2011: 2), existen diversos trabajos que hacen referencia a la competencia digital "referida a la capacidad, conocimiento y actitud de uso de tecnologías de la información y la comunicación en sus diversas funciones y contextos de aplicación". Así, no basta con que los docentes sean competentes en su materia educativa, sino también que lo sean digitalmente o tecnológicamente. Para analizar las competencias TIC se utilizan distintos estándares. Prendes y Gutiérrez (2011: 2), consideran que "un estándar sobre competencias TIC de los docentes puede definirse como un patrón o modelo de referencia que permite, por una parte determinar y valorar a aquellos docentes que son competentes y por otra parte orientar y guiar el diseño y elaboración de las propuestas de formación docente respecto a estas tecnologías". Y según el país o región del mundo se utilizan unos estándares u otros. En Estados Unidos es elaborado por la Sociedad Internacional para la Tecnología en Educación (ISTE). En Europa, UNESCO ha elaborado distintos proyectos donde ofrecen a los docentes orientaciones para que puedan plantear programas de formación. El Proyecto de Estándares de competencia en TIC para docentes (UNESCO, 2008: 7) destaca la idea de que: "las nuevas tecnologías (TIC) exigen que los docentes desempeñen nuevas funciones y también, requieren nuevas pedagogías y nuevos planteamientos en la formación docente. Lograr la integración de las TIC en el aula dependerá de la capacidad de los maestros para estructurar el ambiente de aprendizaje de forma no tradicional, fusionar las TIC con nuevas pedagogías y fomentar clases dinámicas en el plano social, estimulando la interacción cooperativa, el aprendizaje colaborativo y el trabajo en grupo". Así, es función del profesorado renovar su pedagogía incorporando las TIC al aula, de una manera controlada, didáctica y favoreciendo el aprendizaje cooperativo, el trabajo en grupo y la interacción entre los alumnos.

\section{Caso práctico}

\section{Antecedentes}

Conociendo la realidad TIC que tan presente se encuentra en nuestra sociedad, se creyó necesario realizar un cuestionario a una serie de profesores, para valorar la formación que dicho profesorado tenía en relación a las TIC y la valoración que hacían de ellas, así como si propiciaban su uso o no en sus clases con sus alumnos. Aunque el estudio realizado fue muy pequeño se lograron sacar conclusiones muy interesantes. 


\section{Procedimiento}

Se escogió un Instituto de enseñanza secundaria (IES) de la Comunidad Autónoma Canaria. Al grupo de profesores escogidos se procedió a pasarles un cuestionario que pueden observar en el anexo. Es un cuestionario sencillo obtenido de la web: profes.net, que consta de 14 preguntas. Dichas preguntas abarcan desde la frecuencia con la que los profesores se conectan a Internet, para qué utilizan el ordenador, las dificultades que tienen para poder incorporar las TIC en su aula, si utilizan las TIC en su asignatura, qué procesos de enseñanza-aprendizaje creen que aportan las TIC, etc. También se les pide que valoren la formación que han recibido en los últimos años en materia TIC.

El cuestionario lo pudieron realizar tanto en el instituto como en casa y se dio una fecha tope para que fueran entregados. Cuando todos los cuestionarios fueron recogidos se procedió a su vaciado y redacción de resultados. El programa escogido para el vaciado de los datos fue el SPSS, versión 19.

\section{Características de la muestra}

Se escogió un instituto donde aún no estaba implantado el proyecto TIC por parte del centro, por lo que aún no contaban con una plataforma propia, aunque desde la Consejería de Educación del Gobierno de Canarias podían acceder a la plataforma Moodle desde la que crear cursos y apoyar la enseñanza presencial dada en el aula.

Los profesores encuestados fueron un total de 42. De los 42, 27 fueron mujeres y 15 hombres. Todos ellos con licenciatura y dos de ellos, además, doctores. Son personas pertenecientes a la clase media, con puesto fijo en la Consejería y formación complementaria.

\section{Resultados}

Tras la recogida de los cuestionarios se procedió al vaciado de los resultados. Los resultados obtenidos fueron los siguientes:

Todos los profesores suelen conectarse diariamente a internet y utilizan el ordenador para trabajar, los cuales son recursos que tienen en casa, pero menos del $20 \%$ se conecta al ordenador en el instituto, en la mayoría de los casos porque no poseen los recursos para poder hacerlo. Al 75\% le gustaría poder utilizar las TIC en el aula, pero no saben cómo integrarlas correctamente. Asimismo valoran la formación recibida como suficiente, por lo que no se sienten preparados para dar este paso. Entre las dificultades que encuentran para integrar las TIC en su trabajo diario, el $85 \%$ destaca la escasa disponibilidad de los equipos informáticos, la falta de preparación y el $45 \%$ nombra el incremento del tiempo de dedicación y dado que no poseen ese tiempo, no integran las TIC en el aula. Solo encontramos a 3 profesores que utilizan las TIC en su asignatura, para apoyar la enseñanza presencial dada, el resto desearía poder utilizarlas, pero ya sea por tiempo o por desconocimiento no lo hacen. Para casi todos, las TIC son importantes y consideran que favorecen completamente el proceso de enseñanzaaprendizaje por lo que hay que hacer todo lo posible por integrarlas en la educación.

\section{Conclusiones}

Tras conocer los resultados llegamos a diferentes conclusiones: 
- Los profesores tienen, en su mayoría, formación en materia TIC y utilizan diariamente el ordenador y se conectan a internet, ya sea por motivos profesionales o personales. Pero luego, en el instituto o no tienen tiempo o motivación para utilizar estas herramientas, por lo que nos surge una duda, ¿se debe a una falta de motivación del profesorado o los recursos de los que disponen en el centro no son suficientes, así como el tiempo que pueden dedicarle?

- Los docentes han recibido formación en materia TIC, pero no se sienten lo suficientemente preparados para hacer un uso de ellas y no será por falta de formación, sino quizás por la formación recibida, ya que como decía Cabero (2004), muchas veces la formación ha estado más orientada a los saberes técnicos que a los saberes didácticos, como tendría que ser siempre, ya que al profesor no le interesa conocer los detalles técnicos del programa, sino qué uso educativo puede hacer de él en una clase con sus alumnos.

- Los recursos de los que disponen son escasos. Necesitamos contar con medios mucho más nuevos y que estén disponibles para los profesores, ya que si utilizan un recurso y este le da más problemas que si no lo utilizaran, evidentemente no lo volverán a intentar la segunda vez.

- Los profesores, aparte de la formación recibida, son conscientes de la importancia de las TIC, de integrarlas y trabajar con ellas, ya que forma parte no solo del futuro, sino del presente actual. Es vital recordar que la educación no es cosa solo de los docentes y alumnos, sino de la sociedad en general y de la administración educativa en particular, por lo que es esta la que debe hacerse cargo también de que los centros posean recursos que puedan utilizar con sus alumnos e integrar la clase en la sociedad 2.0, así como proveer a los profesores del tiempo y formación para hacer un buen uso de las TIC.

\section{ANEXO: ESTUDIO SOBRE TECNOLOGÍAS EN LA EDUCACIÓN}

Esta encuesta forma parte de una investigación que lleva a cabo un equipo interdisciplinar desde tres ámbitos muy diferentes: el académico ( La Salle-UAM ), el de contenidos (SM-Profes.net) y el tecnológico (Toshiba, NTS). El objetivo es identificar los factores críticos necesarios para una incorporación eficaz de las Tecnologías de la Información y de la Comunicación (TIC) en los centros escolares.

Su opinión es esencial para nosotros, por lo que le rogamos que dedique unos minutos a cumplimentar este formulario. ¡Gracias por su tiempo!

El cuestionario es anónimo, pero si desea que le informemos de los resultados, puede indicar un correo de contacto al final del formulario.

1. ¿Dónde accede a los recursos informáticos habitualmente? (Señale las dos opciones principales)

En casa

En el aula de alumnos

En la sala de profesores

En el aula de informática

Otros: 


\begin{tabular}{|c|c|c|c|c|}
\hline \multicolumn{5}{|c|}{$\begin{array}{l}\text { 2. Valore la frecuencia con la que usa los siguientes programas: } \\
\text { (Marque con una } X \text { sabiendo que } 1: \text { nada y } 4: \text { mucho) }\end{array}$} \\
\hline & 1 & 2 & 3 & 4 \\
\hline $\begin{array}{l}\text { Procesador de texto (Word, Amipro, AbiWord, } \\
\text { etc.) }\end{array}$ & ○ & ○ & ○ & O \\
\hline $\begin{array}{l}\text { Programa de presentaciones (Power Point, Corel, } \\
\text { Presentation, etc.) }\end{array}$ & ○ & O & O & O \\
\hline Bases de datos (Access, MySQL, FileMaker, etc.) & O & O & O & O \\
\hline Hojas de cálculo (Excel, Calc, etc.) & O & O & O & O \\
\hline Navegadores & O & O & O & O \\
\hline Correo electrónico & O & O & O & O \\
\hline Editores HTML (Frontpage, Dreamweaver, etc.) & O & O & O & O \\
\hline Diseño gráfico & O & O & O & O \\
\hline Chat & O & 0 & 0 & O \\
\hline $\begin{array}{l}\text { Herramientas para el trabajo colaborativo } \\
\text { (BSCW, etc.) }\end{array}$ & ○ & ○ & ○ & O \\
\hline Motores de búsqueda & O & O & O & 0 \\
\hline
\end{tabular}

\begin{tabular}{|l|l|}
\hline 3. & Usa el ordenador básicamente para: (Señale las tres opciones principales) \\
\hline$\square \quad$ Formación y perfeccionamiento \\
\hline$\square \quad$ Edición de documentos \\
\hline$\square \quad$ Enseñanza en el aula \\
\hline$\square \quad$ Comunicación con otras personas \\
\hline Otros: \\
\hline
\end{tabular}




\begin{tabular}{|l|l|}
$\begin{array}{l}\text { 4. Las dificultades que encuentra para incorporar la herramienta informática a su } \\
\text { trabajo diario se deben } \mathbf{a :}\end{array}$ \\
\hline$\square \quad$ Falta de preparación \\
\hline$\square \quad$ Incremento del tiempo de dedicación \\
\hline$\square \quad$ Escasa disponibilidad de equipos informáticos en el centro \\
\hline$\square \quad$ Escasa disponibilidad de equipos informáticos en los hogares \\
\hline$\square \quad$ Poca aceptación de la metodología en las familias \\
\hline$\square \quad$ Escasez de materiales didácticos \\
\hline$\square \quad$ Poca adaptación de los materiales al currículo \\
\hline$\square \quad$ Ninguna \\
\hline Otras: \\
\hline
\end{tabular}

\begin{tabular}{|c|c|}
\hline & $\begin{array}{l}\text { Valore la formación en el uso de las Tecnologías de la Información y la Comunicación } \\
\text { TIC) que ha recibido a lo largo de su labor profesional: } \\
\text { Señale el número sabiendo que 1: insuficiente y 4: óptima) }\end{array}$ \\
\hline & 10 \\
\hline & 20 \\
\hline & 3.0 \\
\hline & 40 \\
\hline
\end{tabular}

\begin{tabular}{|l|}
\hline 6. En la(s) asignatura(s) que imparte ¿utiliza las TIC? \\
\hline Sí $\bigcirc \quad$ NO $\bigcirc$ \\
\hline Si la respuesta es afirmativa, señale la frecuencia con que utiliza las TIC en el aula: \\
\hline$\bigcirc$ Diaria \\
\hline$\bigcirc$ Semanal \\
\hline$\bigcirc$ Mensual \\
\hline$\bigcirc$ Trimestral \\
\hline$\bigcirc$ No las utilizo \\
\hline Si la respuesta es negativa, especifique las razones: \\
\hline
\end{tabular}

\begin{tabular}{|l|}
\hline $\begin{array}{l}\text { 7. ¿Considera que las TIC pueden ser un recurso importante para mejorar la en- } \\
\text { señanza? }\end{array}$ \\
\hline Sí $\bigcirc \quad$ NO $\bigcirc$ \\
\hline
\end{tabular}




\begin{tabular}{|c|c|c|c|c|}
\hline $\begin{array}{l}\text { 8. Valore en qué medida las características de las TIC q } \\
\text { pueden favorecer los procesos de enseñanza y aprendiz } \\
\text { que 1: nada y } 4 \text { : mucho) }\end{array}$ & $\begin{array}{l}\text { le se } \mathbf{1} \\
\text { je: }(1\end{array}$ & $\begin{array}{l}\text { encio } \\
\text { arque }\end{array}$ & n a conti & $\begin{array}{l}\text { nuación } \\
\text { abiendo }\end{array}$ \\
\hline & $\begin{array}{c}1 \\
\text { nada }\end{array}$ & $\begin{array}{c}2 \\
\text { poco }\end{array}$ & $\begin{array}{c}3 \\
\text { bastante }\end{array}$ & $\begin{array}{c}4 \\
\text { mucho }\end{array}$ \\
\hline Interactividad & O & O & O & O \\
\hline Individualización de la enseñanza & O & O & O & O \\
\hline $\begin{array}{l}\text { Variedad de códigos de información (texto, sonido, imá- } \\
\text { genes, etc) }\end{array}$ & O & ○ & O & O \\
\hline Aprendizaje cooperativo & O & O & O & O \\
\hline Aprendizaje autónomo & O & O & O & O \\
\hline Alta motivación & O & O & O & O \\
\hline Facilidad de uso & O & O & O & O \\
\hline Flexibilidad para actualizar información & O & O & O & O \\
\hline
\end{tabular}

\begin{tabular}{|ll|}
\hline 9. & Considera que el uso del ordenador es: (Señale los adjetivos que crea oportunos) \\
\hline$\square$ & Entretenido \\
\hline$\square$ & Rígido \\
\hline$\square$ & Manejable \\
\hline$\square$ & Innecesario \\
\hline$\square$ & Agradable \\
\hline$\square$ & Eficaz \\
\hline$\square$ & Complicado \\
\hline$\square$ & Educativo \\
\hline$\square$ & Práctico \\
\hline$\square$ & Importante \\
\hline$\square$ & Perjudicial \\
\hline
\end{tabular}




\begin{tabular}{|l|l|}
\hline $\begin{array}{l}\text { 10. ¿En qué aspectos le gustaría que la tecnología le ayudara en el aula? } \\
\text { (Señale las dos opciones principales) }\end{array}$ \\
\hline$\square \quad$ La obtención de materiales didácticos \\
\hline$\square \quad$ La atención a la diversidad \\
\hline$\square \quad$ La comunicación con los padres \\
\hline$\square \quad$ El refuerzo de contenidos básicos \\
\hline$\square \quad$ El mantenimiento de la disciplina en el aula \\
\hline$\square \quad$ El tratamiento individualizado de los alumnos \\
\hline$\square \quad$ La mejora de la atención en clase \\
\hline$\square \quad$ La motivación de los alumnos por la asignatura \\
\hline$\square \quad$ La interdisciplinariedad \\
\hline
\end{tabular}

\begin{tabular}{|l|}
\hline 11.- ¿Cómo cree que debería ser la financiación de los recursos TIC del centro? \\
\hline ○ Pública \\
\hline ○ Privada \\
\hline ○ Convenio (empresas, universidades...) \\
\hline ○ Donación (bancos, fundaciones...) \\
\hline
\end{tabular}

\begin{tabular}{l}
$\begin{array}{l}\text { 12.- ¿Existe un plan estratégico por escrito para la incorporación de las TIC en su } \\
\text { centro? }\end{array}$ \\
\hline SÍ $\bigcirc \quad$ NO $\bigcirc$ \\
\hline
\end{tabular}

13.- En caso de existir el plan anteriormente mencionado, ¿lo conoce el profesorado? SÍ $\bigcirc \quad$ NO $\bigcirc$ 
14.- Valore los siguientes factores en función de su importancia de cara al éxito de la implantación de las TIC en el centro.

(Marque con una X sabiendo que 1: poco importante y 4: muy importante.)

\begin{tabular}{|c|c|c|c|c|}
\hline & 1 & 2 & 3 & 4 \\
\hline Número de ordenadores & O & O & O & O \\
\hline Número de profesores participantes & O & O & O & O \\
\hline Número de asignaturas implicadas & O & O & O & O \\
\hline Aceptación de las familias & O & O & O & O \\
\hline Aceptación de los alumnos & O & O & O & O \\
\hline Aceptación de los profesores & $\mathrm{O}$ & O & O & O \\
\hline Resultados académicos & O & O & O & O \\
\hline
\end{tabular}

\begin{tabular}{|l|}
\hline * Datos de clasificación (anónimos) \\
\hline 1.- Edad \\
\hline O Entre 21 y 30 \\
\hline ○ Entre 31 y 40 \\
\hline O Entre 41 y 50 \\
\hline O Más de 50 años \\
\hline
\end{tabular}

\begin{tabular}{|l|}
\hline 2.- Titulación \\
\hline O Científica - Tecnológica \\
\hline O Humanidades \\
\hline Biosanitaria \\
\hline$\bigcirc$ Otros \\
\hline
\end{tabular}

\begin{tabular}{|l|}
\hline 3.- Años de ejercicio docente \\
\hline$\bigcirc$ De 1 a 5 \\
\hline De 1 a 5 \\
\hline De 6 a 10 \\
\hline De 11 a 15 \\
\hline De 16 a 20 \\
\hline De 21 a 25 \\
\hline Más de 25 \\
\hline
\end{tabular}




\begin{tabular}{|l|}
\hline 4.- Categoría \\
\hline$\bigcirc$ Profesor \\
\hline$\bigcirc$ Coordinador TIC \\
\hline$\bigcirc$ Jefe de Estudios o Coordinador \\
\hline$\bigcirc$ Director \\
\hline$\bigcirc$ Equipo de dirección \\
\hline$\bigcirc$ Otros cargos \\
\hline
\end{tabular}




\section{Referencias}

Barberá, E. (2003). La educación en la red. Actividades virtuales de enseñanza y aprendizaje., Barcelona: Paidos.

Cabero, J. (2004). Formación del profesorado en TIC. El gran caballo de batalla. Comunicación y Pedagogía. Tecnologías y Recursos didácticos, (ISSN 1136-7733).195, 2004, 27-31). Recuperado el 21 de mayo de 2012 de la siguiente dirección web: http://tecnologiaedu.us.es/cuestionario/bibliovir/jca11.pdf

Cebrián de la Cerna, M. (2003). Innovar con tecnologías aplicadas a la docencia universitaria, en CEBRIÁN DE LA SERNA, M. (coord): Enseñanza virtual para la innovación universitaria, Madrid: Narcea, pp. 21-36.

Escudero, J. (2006). El profesor y su formación: Competencias y formación docente al servicio de un modelo de escuela en nuestro tiempo. En Programa y desarrollo temático de Formación y Actualización de la Función Pedagógica (pp. 84-108). Universidad de Murcia.

Gutiérrez, I. y Prendes, M. (2011). Competencias tecnológicas del profesorado en las Universidades Españolas. Revista de Educación, 361. Consultado el 20 de mayo de 2012 de la siguiente dirección web: http://www.revistaeducacion.mec.es/doi/361_140.pdf

UNESCO (2008). Estándares de competencias en TIC para docentes. Recuperado el 21 de mayo de 2012, de http://www.eduteka.org/modulos/11/342/868/1

\section{Sobre el Autor}

Paula Morales Almeida: Soy educadora social y psicopedagoga. Actualmente me encuentro realizando el doctorado en Formación del Profesorado por la Universidad de Las Palmas de Gran Canaria. Mi línea de investigación son las TIC y educación, es por ello que me gusta investigar sobre lo que las TIC pueden ofrecernos educativamente hablando. Mi tesis va orientada hacia la formación del profesorado de adultos en TIC y es un trabajo que me está aportando muchas cosas; información, formación, aprendizaje, etc. He participado en los congresos de Málaga: Usos y Buenas prácticas con TIC, celebrado en diciembre de 2011 y en las III Jornadas Internacionales de Mayores y nuevas tecnologías, celebrado en Castellón en abril de 2012. Me gustaría seguir ligada a la investigación educativa, especialmente en la formación del profesorado y en las TIC. 